\title{
"Y'a toujours à penser, à trouver mieux". Moments effondristes et activisme du quotidien face à la catastrophe écologique
}

\author{
Alexandra Bidet (https://orcid.org/0000-0003-4470-9831), \\ Centre Maurice Halbwachs, Centre National \\ de la Recherche Scientifique, Paris, França!.
}

Solène Sarnowski (https://orcid.org/0000-0003-4470-9831), École des Hautes Études en Sciences Sociales, Paris, França".
Recebido: 18.04 .21 Aprovado: 26.07 .21

\author{
I. Alexandra Bidet \\ é professora \\ de sociologia e \\ pesquisadora no \\ Centre Maurice \\ Halbwachs (CNRS- \\ EHESS-ENS), \\ Centre National \\ de la Recherche \\ Scientifique (CNRS), \\ Paris, França. \\ <alexandra.bidet@ \\ gmail.com>. \\ II. Solène Sarnowski \\ é mestranda na École \\ des Hautes Études \\ en Sciences Sociales, \\ Paris, França. \\ <solene35. \\ sarnowski@gmail. \\ com>.
}

Résumé: Face à la catastrophe écologique, de nouvelles formes d'engagement apparaissent, qui font la part belle à l'enquête. A partir d'entretiens menés avec des personnes engagées dans la sensibilisation aux enjeux écologiques, nous mettons ainsi en évidence un activisme du quotidien, dont les protagonistes soumettent à une enquête permanente leurs habitudes de vie, mais aussi le crible même de leurs enquêtes: ce qu'écologique veut dire. Un tel engagement contrarie la dissociation commune entre éthique et politique. Les enquêtes de ces activistes, portées par une passion plus pratique que cognitive, sont en effet aussi habitées par l'horizon d'un monde commun et de la production d'irréversibilités au sein de nos formes de vie.

Mots-clés: Écologie. Enquête. Engagement. Citoyenneté. Habitudes.

\section{"Há sempre algo para se pensar ou fazer de melhor". Momentos de colapso e ativismo cotidiano diante de uma catástrofe ecológica}

Resumo: Diante do desastre ecológico, novas formas de engajamento que dão lugar de destaque à investigação estão surgindo. A partir de entrevistas realizadas com pessoas envolvidas na sensibilização para as questões ecológicas, destacamos assim um ativismo cotidiano, cujos protagonistas submetem seus estilos de vida a um levantamento permanente, mas também ao próprio crivo de seus levantamentos: este que é ecológico. Esse compromisso impede a dissociação comum entre ética e política. As investigações desses ativistas, movidas por uma paixão mais prática do que cognitiva, são, de fato, habitadas também pelo horizonte de um mundo comum e pela produção de irreversibilidades dentro de nossas formas de vida.

Palavras-chave: Ecologia. Pesquisa. Engajamento. Cidadania. Hábitos. 
1. Le terme apparaît avec l'ouvrage en 2015 de P. Servigne et R. Stevens,

Comment tout peut s'effondrer. Mais L. Semal rappelle que ce thème contemporain prolonge "une inquiétude existentielle propre à l'écologie politique depuis ses origines: le déclin ou l'effondrement de la civilisation thermo-industrielle comme conséquence possible, probable ou certaine de la catastrophe écologique globale déjà amorcée" (Semal, 2019b).

\section{Entretien avec}

A. Damasio, "Sans renouement avec le vivant, il n'y a pas de sortie du technocapitalisme", Reporterre, 2 mai 2019.

3. "Appels sans suite (1)", Blog. mondediplo.net, 12 octobre 2018.

\section{F. Thoreau et B.} Zitouni, "Contre l'effondrement: agir pour des milieux vivaces", Lundimatin, 19 décembre 2018.

5. Sans souci d'inventaire exhaustif, mentionnons en quelquesunes: le concept d'effondrement est "particulièrement pauvre en termes critiques", estime la sociologue $\mathrm{E}$. Lagasse in "Contre l'effondrement, pour une pensée radicale des possibles",

\section{"You can always think further, find something better" Collapsing moments and day-to-day activism in the face of the ecological disaster}

Abstract: In front of the ecological disaster, new forms of engagement give great importance to the practice of inquiring. Based on interviews conducted with people involved in raising awareness on ecological issues, we thus highlight a day-to-day activism, whose protagonists subject their lifestyles to a permanent scrutinity, but also the very criterion of their valuations: what ecology means. Such a commitment thwarts the common dissociation between ethics and politics. The investigations of these activists, driven by a more practical than cognitive passion, are indeed also inhabited by the horizon of a common world and the production of irreversibilities within our forms of life.

Keywords: Ecology. Investigation. Commitment. Citizenship. Habits.

\author{
"Le manque criant ne se manifeste pas \\ tant au niveau de la volonté d'agir en \\ fonction de biens déjà connus que dans la \\ volonté de savoir ce qu'ils sont". \\ (John Dewey, 2014: 284).
}

J idée d'effondrement, associée depuis le milieu des années 2010 à la "collapsologie"1, "compilation transdisciplinaire" (Cravatte, 2019) alertant sur les risques du franchissement des "limites planétaires", est spontanément tenue pour démobilisatrice, sinon dépolitisante. "Il y a une énorme complaisance à dire qu'on va droit dans le mur. Je ne trouve pas cela mobilisant", juge A. Damasio². F. Lordon a tourné en dérision les appels pour le climat, en écrivant qu'ils veulent "avertir de la fin des temps pour exiger la fin des touillettes"3. F. Moreau et B. Zitouni tiennent l'idée d'effondrement pour "le plus mauvais service que l'on puisse rendre aux gens et aux collectifs en lutte contre les ravages environnementaux" ${ }^{4}$. Et de résumer le verdict en estimant que privilégier une "pensée de la rupture" sur une compréhension des continuités fabrique "des êtres nus, arrachés à ce qui les tient et à ce qui leur importe [...] des citoyens ignorants et désemparés", ayant "tout perdu", "sans peuples et sans devenirs particuliers" (ibidem). Les prises de position en ce sens ont été abondantes ${ }^{5}$. Toutes supputent les effets d'une notion sans en explorer les usages ni les espaces de circulation. Ainsi, les critiques de "l'effondrisme" laissent les "publics de l'effondrement" à l'état de "fantômes" (Allard, Monnin \& Tasset, 2019: 59). Elles ne soumettent pas à l'enquête l'idée que les "effondrés" se contentent d' "attendre l'effondrement du capitalisme fossile", comme 
l'écrit J.-B. Fressoz, ou que "collapsos et preppers ne se préparent à rien et ne préparent pas grand-chose", comme le soutient F. Tison.

Or les premiers travaux concernant ces publics (Allard, 2019; Chamel, 2019; Semal, 2019a; Tasset, 2019) suggèrent non seulement que l'effroi catastrophiste n'empêche nullement les militants de s'engager (Semal, 2019a), mais aussi que des "formes collectives hésitantes" se constituent bel et bien "autour de la conviction catastrophiste" (Tasset, 2019). Explorer "l'effondrement vu d'en bas", à travers ce "que fait l'effondrement, et ce qu'en font celles et ceux à qui cette idée fait quelque chose" (Allard, Monnin \& Tasset, 2019), c'est frayer une posture a contrario pragmatiste, qui réinscrit les idées dans le monde pour en explorer la portée, et piste les visées et les idéaux in the making en suivant la "fabrique pratique de problèmes amis", où se fabule "la réalité du possible", selon les formules de J. Rafanell i Orra (2018: 82). Les échanges au sein de groupes Facebook organisés autour de la collapsologie (notamment Transition 2030, Coming-out effondrement, La collapso heureuse etc.), en forte croissance numérique depuis l'été 2018, font bien de l'effondrement un problème sans cesse requalifié, qui subit un net glissement quand l'expérience associée ne consiste plus tant à s'approprier ni à synthétiser un corpus de connaissances, qu'à faire avec le surgissement d'un monde où ce qui faisait milieu s'est défait; car, suivant l'expression de B. Latour, il n'y a plus de terre où atterrir (2017). L'effondrement n'est alors plus tant une catastrophe qu'on guette, redoute ou appelle, que le fil d'Ariane d'un concernement qui éprouve et fait faire, c'est-à-dire aussi défaire. Que ce terme cristallise le caractère "irrévocable" ${ }^{6} \mathrm{~d}^{\prime}$ une préoccupation, ne signifie pas qu'il détourne des choix relatifs aux "conditions matérielles existantes", comme le redoute J. Cravatte (2019). La nécessité soudaine, pour les "effondrés", d'explorer les relations situées qui les font exister radicalise au contraire volontiers l'attention qu'ils leur portent. Les participants de ces groupes multiplient les enquêtes face à des questions urgentes et intriquées: quoi faire, où habiter, avec qui, comment s'associer, où se rencontrer etc.? La diffusion accélérée du vocable d'effondrement témoignerait donc de la multiplication des enquêtes pour trouver une terre où atterrir (Bidet, 2019), plus que de la force intrinsèque d'une idée ou d'une promesse (Charbonnier, 2019). L'importance des échanges et du partage d'expériences en ligne et hors ligne s'éclaire aussi: enquêter intensément sur ce qui vaut suppose la production continuée d'un "sentiment de confiance", pour pouvoir passer outre la quête de certitudes (Dewey, 2014: 26).

Afin de contribuer à explorer les formes d'engagement de "publics" de l'effondrement, et la portée de "moments effondristes" au gré de trajectoires ne passant pas forcément par des associations ou des groupes se revendiquant tels, nous allons nous pencher, sans souci de représentativité, sur l'expérience de neuf personnes paru le 18 juillet 2018 dans la revue Contretemps; la philosophe E. Hache, in "Écologie politique et écoféminisme", tient l'effondrement pour "une façon très dépolitisante de poser le problème", Présages, 14;

I'historien des sciences J.-B. Fressoz, "La collapsologie: un discours réactionnaire?", paru dans Libération le 7 novembre 2018, voit dans "la vogue de l'effondrement" le risque de "négliger la dimension politique des enjeux écologiques"; il est question de "résignation fataliste" dans la contribution de D. Tanuro,

"L'effondrement des sociétés humaines est-il inévitable? Une critique de la 'collapsologie': c'est la lutte qui est à l'ordre du jour, pas la résignation endeuillée", Gauche Anticapitaliste, 27 mars 2018, repris sur Contretemps, le 19 juin 2018; F. Tison estime que l'accent mis sur l'effondrement à venir, plutôt que sur la catastrophe en cours, "ne laisse pas beaucoup d'autres perspectives qu'une nouvelle forme de passivité, fébrile et repliée, même si elle invite à autre chose", in "Redéfinir la situation - Notes sur la collapsologie et son impact", Lundimatin, 18 juillet 2019; le philosophe B. Bégout voit dans ce qu'il qualifie de "sophistique 
permanente de la catastrophe" un ferment de "pure et simple résignation", in "L'anti-évangile de notre temps", Junkpage, juillet-août 2019; dans le dossier "Comment penser l'effondrement?" de la revue Dard/ dard, v. 2, n. 2, 2019, le philosophe P.-H. Castel stigmatise "la futurologie des catastrophes", tout en considérant qu'il "est vraisemblable que le monde va s'effondrer dans un certain ordre", et que "c'est même toute idée classique d'action et d'action collective qui s'effondre du même mouvement synchrone que les écosystèmes qui lui avaient aimablement, jusqu'ici, prêté leur fidèle support". Enfin, dans leur ouvrage Le pire n'est pas certain. Essai sur l'aveuglement catastrophiste, en septembre 2020, C. et R. Larrère opposent les réactions supposément privées et dépolitisées des collapsologues à ce qui relèverait de luttes locales autour des milieux de vie.

6. "Irrévocablement, s'empare alors de vous la révélation que jamais plus vous ne serez celui que vous avez été" (Fitzgerald, 1936).

7. Au moment des entretiens, trois enquêté.e.s sont membres de Citoyens Pour Le Climat, une est bénévole à la engagées, ou aspirant à s'engager, dans la sensibilisation aux enjeux écologiques. Afin de diversifier les profils et de ne pas partir d'une catégorie prédéfinie, leur recrutement s'est fait au fil des interactions permises par la présence de l'une d'entre nous à des actions écologiques ouvertes à tous: le blocage des ponts mené à Paris en octobre 2019 par l'association Extinction Rébellion, une conférence organisée le même mois à l'Université de Nanterre sur le thème "Que faire pour la planète?" et des Marches pour le climat. Parmi les enquêté.e.s rencontrés, six sont engagés dans divers collectifs ou associations ${ }^{7}$, depuis quelques semaines ou plusieurs années. Trois personnes manipulent de très près les prévisions du GIEC (Groupe d'Experts Intergouvernemental sur l'Étude du Climat), car elles participent à des activités de vulgarisation et de sensibilisation aux enjeux climatiques. Âgés de 17 à 53 ans, ces enquêté.e.s comptent six femmes et trois hommes. Huit habitent en région parisienne, dans des conditions allant de la maison familiale périurbaine entourée d'un grand jardin au petit appartement parisien, et du périurbain ou de la proche banlieue aux denses 10 e et 12e arrondissements, en passant par les banlieues du sud et de l'ouest parisien. Tout.e.s n'ont pas toujours habité en ville. Parmi les six enquêté.e.s en couple, cinq vivent avec leur partenaire, l'un a trois enfants, et la plus jeune habite encore le foyer parental. Malgré des situations professionnelles hétérogènes (agricultrice, isolateur, ingénieur aéronautique et designer écologique, pour ceux qui ne sont plus étudiants), le niveau de diplôme, au moins Master, est plutôt homogène.

Présenter les aspects partagés de leurs expériences va nous conduire à thématiser un activisme du quotidien, où les nombreuses initiatives prises pour transformer sa façon de vivre peuvent se mêler, sans solution de continuité ni hiérarchie, à des actions de rue et/ou à des actions de sensibilisation, diversement inscrites dans des initiatives collectives et/ou des collectifs, en brouillant les frontières entre les espaces d'activité. Au lieu de se définir à partir de la scène militante ou même associative (Ollitrault, 2009), cet activisme s'ancre dans la vie quotidienne, où tous décrivent une "transformation douce" de leurs habitudes de vie au "crible" de l'écologie - un crible divers et mouvant, lui-même soumis à une enquête permanente: "y'a toujours à penser, à trouver mieux". Tout en partageant l'orientation vers le "faire autrement" d'autres publics de l'écologie, à l'instar des écologistes radicaux étudiés par B. Mège (2017) et G. Pruvost (2013), "véritables enquêteurs" développant des savoir-faire "impliquant une succession d'essais et d'erreurs", ils s'en distinguent toutefois. Car c'est ici dans un contexte plus ordinaire que s'observe une trajectoire de "politisation du moindre geste" que G. Pruvost (2015) a étudié pour sa part à travers "I'expérience politique totale" de la participation à un chantier participatif. Les activistes du quotidien considérés ici n'ont pas réorienté leurs activités autour de l'autoproduction, vivent pour la plupart en ville, et fréquentent des 
milieux de travail ou d'éducation supérieure encore peu investis par des projets de "redirection écologique" (sur l'exemple du design: Monnin \& Allard, 2020; Bonnet et al., 2019). L'inconfort de leur position frontière, située entre le tout-venant et les "écologistes radicaux" - et investie comme telle d'une visée de sensibilisation, va apparaître à la mesure de leurs efforts pour travailler leur propre plasticité et, indirectement aussi, celle des autres.

Partir de leurs moments effondristes va nous conduire à préciser la singularité des enquêtes qu'ils mènent (1), ce qu'elles leur font (2), le type de radicalité que leurs pratiques déploient (3) et la façon dont des irréversibilités s'y cherchent à I'horizon d'un monde commun (4).

\section{Passion cognitive ou passion pratique?}

Aucun des enquêté.e.s ne se dit "effondriste" ou "collapso"; le terme d'effondrement est néanmoins présent dans un entretien, celui de "collapsologie" dans un autre, et la référence à Pablo Servigne dans un troisième. Et tous font état d'un horizon catastrophique: un "énorme problème", une "menace ultime", la perspective du "pire futur", un "génocide", "y’a tout qui va craquer", "c'est la merde" etc. Des "chiffres graves" les ont "assis": "j'étais jamais allé voir dans les chiffres, et quand tu vas voir les courbes principales issues du GIEC, y'en a une qui te met une grand claque dans la tête, en disant que pour l'instant on a pas du tout varié de trajectoire, et si on ne varie pas du tout de trajectoire, la courbe d'émission de gaz à effet de serre se met à stagner autour de 2080... la grosse rouge ouais, celle qui stagne parce qu'on tue des centaines de millions de personnes". Ils narrent des périodes où ils se sont "plongés dans les chiffres à fond", comme des moments plus ponctuels où ils vont "voir dans les chiffres", pour "creuser" une question ou trancher un différend ("sujet classé"): "[mon mari] n'avait pas les chiffres, il ne pouvait pas comprendre". Ils thématisent aussi l'idée d'effondrement à travers l'évocation de civilisations passées, l'image du "mur", ou de façon plus diffuse, à travers un horizon de catastrophes, la récurrence d'alertes, la mise en danger de l'espèce humaine ou du vivant, ou encore le surgissement d'un sentiment de vulnérabilité qui est tout autant personnel qu' "historique", comme en témoigne ce parallèle fait avec les attentats parisiens de 2015:

Après les attentats... Pendant longtemps, tu prends le métro et tu te dis 'ah ouais, je ne suis pas en sécurité en fait'. Et là pareil, j'ai l'impression de participer à une période de changement historique, où y'a des changements de pratiques, et cet instinct de
Bascule, une à La Base et un aux Amis de la Terre. Plusieurs participent à des actions d'Extinction Rébellion. 
survie surtout je pense. Clairement, je sens que l'espèce est en danger, moi je sens au plus profond de moi-même que notre espèce est en danger.

L'attention ainsi prêtée aux chiffres, et l'expérience associée au défaut de terre "où atterrir", n'impliquent pas un rapport scientiste à l'idée d'effondrement, que l'on entende par là un évènement brutal ou bien un enchaînement graduel, multiforme, voire même imperceptible, de phénomènes aux temporalités disparates, dont les mécanismes pourraient être tracés à l'avance. Jusque sur les forums "effondristes", le vocable d'effondrement se voit d'ailleurs reprocher son "flou", son simplisme, son côté attrape-tout - quand il n'est pas évalué à sa seule portée rhétorique par ses promoteurs mêmes. Or ni les effets de cette notion ni sa prétention à totaliser des savoirs ne semblent vraiment préoccuper nos enquêté.e.s. L'effondrisme comme "passion cognitive", analysé par C. Tasset (2020) comme une expérience intellectuelle d'autant plus intense qu'elle imbrique "I'annonce d'une débâcle et la conquête d'un savoir" , n'est pas au cœur de leurs récits. Quand ils narrent des périodes de visionnage intensif de vidéos Youtube ou d'autres ressources en ligne, c'est à la manière d'une activité ponctuelle, ouvrant sur une révision de leurs habitudes de vie, plus que sur la relance indéfinie d'une entreprise de totalisation intellectuelle:

En faisant ce travail de vulgarisation, j'ai appris plein de choses, j'ai acquis une vision un peu plus globale du problème, et bah là quand tu vois l'urgence, tout de suite, au niveau des pratiques individuelles, ça impactait, au premier janvier donc on est devenu vraiment végétariens, enfin flexitariens;

Le climat, par rapport à la biodiversité, comme je m'y connais un peu moins, j'ai moins tendance à changer mes habitudes pour cela: je le fais quand même, mais moins.

L'accumulation de connaissances passe, elle-même, autant par des explorations pratiques. Ainsi, pour cette enquêtée lors de son long séjour à Montréal: "C'est une période où j'ai découvert la permaculture, le zéro déchet, le végétarisme, le véganisme, c'est là aussi où je me suis intéressée à d'autres formes d'agricultures, l'agriculture urbaine, l'aquaponie, enfin plein de choses... du coup c'était, ouais, une espèce d'émulation intellectuelle parce que je me renseignais, j'acquérais des connaissances etc., autant des conférences que de mes cours, mais aussi de la mise en pratique directe". De même, l'agencement de ressources en ligne - série de blogs ou d'abonnements Instagram, vise avant tout à entretenir une dynamique d'action(s), à répondre à un intense "besoin d'agir": "Je n'étais pas à fond. J'étais abonnée à quelques trucs sur Insta, maintenant je suis abonnée à plein de trucs 
sur Insta. J'ai toujours plein de trucs pour être toujours dans le mood tu vois, pas lâcher, parce qu'avec le rythme de la vie tu peux facilement lâcher prise, mais faut pas lâcher tu vois". La création de liens est une autre médiation très importante ici, à travers aussi bien des "dynamiques de co-information sur ce qui se passe" (entre ami.e.s), qu'un partage des rôles (très fréquent au sein des couples: "elle trouve et j'exécute", "il produit et moi je coordonne", "on se complète, c'est elle qui me motive") ou des formes d'émulation ("ok, ma mère m’a dépassée!"). Le lexique se fait même volontiers militaire pour décrire la mobilisation propre à entretenir cet engagement: "ma bagarre quotidienne", "sur le front", "engagée à 100\%", "à fond dans l'écologie", "participer à l'effort de guerre".

Mais c'est peut-être comme une enquête que cette passion pratique se laisse le mieux décrire, tant son objet reste largement indéterminé, ouvrant sur des finsen-vue sans cesse renouvelées: non pas préparer, précipiter ou éviter "I'effondrement", mais "l'écologie".

\section{Enquêter:}

attester d'un possible, changer de monde

La posture d'enquête se manifeste par des préventions partagées: on se garde de croire que l'on sait définitivement "quoi faire" - il y a toujours des choix pratiques à "creuser", la nécessité de "se renseigner", de "pousser la réflexion"; comme on se refuse à penser que l'on "en fait assez" - on cherche constamment comment en "faire plus", "se démarquer", "progresser", ou bien "se relancer" quand on a l'impression de "stagner" ou d'avoir atteint un "palier". Tous soulignent le caractère "hyper graduel", "progressif", "complexe" ou encore "par petites touches", d'une "transformation douce", qu'ils ressentent néanmoins d'autant plus vivement qu'elle produit au cœur de leurs habitudes les plus quotidiennes des irréversibilités, à l'image de "caps" passés, excluant toute perspective de retour en arrière.

Associées à des tâtonnements, ces irréversibilités sont fort éloignées des "étapes", à la destination fixée d'avance, des visions classiques de l'engagement. "L'engrenage", décrit au sein du quotidien ("une fois que tu changes une brique de ton style de vie, tout arrive, tout change") peut en effet s'y maintenir sans que l'engagement ne perde sa valeur ni sa portée. Les valuations négatives ("I'angoisse", "je me stresse de ouf", "désespérant", "déprimant" etc.) associés à la catastrophe écologique sont aussi nombreuses que les valuations positives ("inouï", "gens incroyables", "répercussions énormes", "super intéressant" etc.), sanctionnant l'évidence de "participer à quelque chose de global", d' "être au cœur du truc": "J'étais 
8. W. James souligne ainsi qu'un choix engage toujours un acte de croyance (Stengers, 2007: 153). tellement enthousiaste dans ce truc-là, et tellement contente d'avoir, tu vois, appris autre chose, et de me dire, mais oui, mais en fait, mais purée, c'est tellement évident, mais c'est hyper satisfaisant dans l'écologie quand t'apprends un petit geste qui en fait a des répercussions énormes". Faire, participer, s'organiser: en même temps que le plaisir pris aux activités, se dit la "sensation agréable que des choses se mettent en place".

Chez ces enquêté.e.s, au niveau d'études élevé, le désir de s'assurer une expérience sensée hic et nunc ne s'épuise nullement dans une totalisation intellectuelle. II s'agit plutôt d'attester d'un possible au présent. Dans une perspective pragmatiste, ce possible est d'autant plus important que les visées n'émergent "qu'en fonction des possibilités propres aux situations dont on a fait l'expérience effective" (Dewey, 2011: 300), dans des moments où "l'imagination idéalisante saisit les choses les plus précieuses rencontrées dans les moments cruciaux de l'expérience et les projette" (Dewey, 2011b: 137). Tout se passe comme si l'exploration pratique des possibles, au ras du quotidien, les faisait "sauter à pieds joints" 8 dans un monde aux transformations désirables. Et ce, par leurs entours, pourrait-on dire, plus qu'en réinvestissant une figure classiquement prométhéenne du geste humain (Flahaut, 2008). L’auto-dérision n'est d'ailleurs jamais loin du désir de "sauver le monde en ramassant des déchets" ou d'être "Alice au pays des merveilles écologiques".

Changer de monde. Leur enquête, en aspirant à se faire systématique, favorise une totalisation de leur existence, que la situation d'entretien ne fait que souligner: "au fond, toute ma vie est liée à la question", "je sais déjà que je passe déjà toutes mes décisions au crible de ces questions-là. Et là, cela ressort d'autant plus quand je t'en parle!". En témoigne l'usage systématique d'expressions ordinairement moins communes: chacun thématise son "mode de vie", son "organisation de vie", son "nouveau style de vie", la façon dont il a "réorganisé" sa vie etc. Et de narrer les différents renoncements, aisés ou difficiles, opérés, en cours, projetés, ou encore peu envisageables - viande, avion, vêtement neuf, emballage, chat, plastique, Netflix, collants, voiture, smartphone, maternité etc. L'impact des habitudes concernées est pointé, comme le trouble sensible associé: "on s'en parlait de passer au vrac, moi je ne pouvais plus voir mes poubelles"; "ça me semblait démentiel d'acheter et jeter des trucs en permanence"; "ça me va pas de déplacer deux tonnes de voiture chaque fois que j'ai besoin de vérifier si la serre s'est pas ouverte ou d'aller fermer l'eau". Les "caps" franchis laissent a posteriori un sentiment d'évidence, même s'ils ont pu être négociés âprement et ne sont pas à l'abri d’aléas. L'inconfort émane aussi d'un processus qui "s'auto-entretient", cette "routinisation de la réflexivité" déjà documentée dans le domaine alimentaire (Halkier, 2009): "on a réfléchi à 
quasiment l'intégralité de nos achats, même le chocolat", "maintenant, tout ce qu'on achète, on réfléchit beaucoup beaucoup beaucoup, peut-être même trop". Pour ne pas devenir invalidante, elle exige de savants dosages, mais aussi de la souplesse et des révisions locales:

Si tu passes vraiment tout le temps tout le temps à te poser des questions, tu t'en sors plus en fait. Et vraiment, on a dû passer des heures à aller, à errer d'un resto à un autre, jusqu'à ce que j'abandonne et que je prenne un truc qui en plus ne me convenait pas en général, et ça a été douloureux, ça a été pendant plusieurs mois, et à un moment, on s'est dit, bon, aller, faut arriver à lâcher prise sur ça parce que là ça nous rend trop malheureux... J'ai dit tant pis sur ce bout-là. Parce que c'était l'angoisse: merde, on va devoir manger dehors [rire]. Sinon cela voulait dire préparer l'intégralité de nos repas, et transporter... ça aurait pu, on l'a fait à un moment, mais c'était devenu... c'est vrai, trop complexe.

Changer de monde. Ce saut engage également le paysage social environnant tant les enquêtes ont leur milieu associé: une "société qui change", des "gens incroyables", "motivés", "super intéressants" etc. Un "nouveau monde" se surimpose à un "monde qui se détruit". L'effet est majoré quand l'activisme, en revêtant une dimension institutionnelle, décuple le champ de vision: "en tant qu'activiste, on est invité dans différents endroits [...] on est quand même aux premières loges, on voit par exemple tous les gens qui se sont engagés, par exemple sur la permaculture, y'a plein de réseaux en lle-de-France, nous on les connaît. Donc en fait, dans notre paysage à nous, on rencontre plein de gens, on discute".

Ces activistes ou aspirants activistes répondent à l'effroi effondriste par un changement de monde constamment cultivé "à l'échelle de la vie quotidienne, dans les instants accordés à la pensée et à l'exploration de multiples attachements personnels (Bidet \& Boutet, 2013). Là où l'horizon pourrait être absorbé par l'anticipation ou la crainte d'un basculement à venir, l'attention est plutôt focalisée sur le présent, dont l'inconfort chronique, entretenu par la propension à sans cesse relancer l'enquête, coexiste avec le constat d'une multitude d'effets heureux. Aux bords de l'irréversible, les irréversibilités gagnent en relief, au point même de pouvoir nourrir des bifurcations professionnelles. La radicalité thématisée par les enquêté.e.s, si elle n'est pas en tant que telle "effondriste", n'est alors pas sans lien avec la "puissance de désertion" de l'effondrisme (Chédin apud Tasset, 2019). 


\section{Radicalité, bifurcations et multi-engagement}

Le thème de la radicalité trame les entretiens: on exige de soi "un minium de radicalité", on se déclare "radicalisé", "devenant radicale", "très radicale dans tout ce que je veux faire" (ne pas avoir d'enfants notamment pour une enquêtée), on juge son conjoint "moins extrême" etc. Le chemin parcouru, ou que l'on projette de parcourir, est marqué par une transformation de soi entretenue et travaillée dans une radicalité de la posture. Entre revendication et prévention du stigmate - tel ce soupçon de pathologie répandu envers les "preppers" (Barker, 2019), les enquêté.e.s laissent la porte ouverte à une distance au rôle quand ils se considèrent "monomaniaque", "obsessionnelle", "psychopathe", "un peu fou", attentif à ne "pas devenir dingue", ou capable de se "déconnecter" ("si je veux, je peux débrancher, aller dans un musée, et je me pose pas la question de savoir si au XV siècle, le pigment...").

Au-delà, ce vocable signale aussi des choix d'orientation professionnelle pour les plus jeunes et de véritables bifurcations, souvent au long cours, pour ceux déjà en activité. La vie laborieuse, comme les études, revêtent des airs d'activisme, quand elles ne s'y confondent pas. Ceux en études (dont deux en Master de "gestion des risques") s'emploient ainsi à aligner leur "choix de métier" sur leur engagement bénévole (diffusion d'une revue de presse de sensibilisation sur son temps libre, organisation de week-ends zéro déchet etc.), quand ceux en activité témoignent de parcours où les réorientations ont largement estompé les frontières entres espaces d'activité.

Une des enquêté.e.s (53 ans), après un début de vie professionnelle dans le numérique, a ainsi réinvesti ses compétences pour développer la présence en ligne de plusieurs grosses ONGs suite à ce qu'elle décrit comme une "grande transformation", qui l'a vue partir en Angleterre pour un Master de design écologique, à la suite duquel elle se réoriente vers l'accompagnement au changement (écologique) des entreprises. La continuité entre vie personnelle et vie professionnelle se donne à lire, au détour d'un banal achat, comme une simple concurrence pratique:

Je cherche des chaussures de qualité en cuir, j'essaye de trouver chaussure à mon pied, je n'y arrive pas, donc je discute avec les gens dans les magasins qui sont responsables des marques, un peu comme une enquête participante, mais émergente, je cherche pas du tout à travailler dans le milieu de la mode! [...]. Les chaussures, elles ont de plus en plus de plastique; là ce n'est pas moi qui change de pratiques, c'est le secteur de l'habillement; 
du coup, ce que je faisais jusqu'à présent se trouve refoulé en pratiques de pointe! [...] Plusieurs fois, je me suis dit: je vais contacter les marques, ça commence vraiment à me saouler... [...] Je n'ai pas eu le temps et puis parce qu'au niveau professionnel, j'ai des leviers, je ne peux pas tout mettre sur les gestes qui sont personnels, parce que j'ai tellement de leviers de changement...

Un autre enquêté (43 ans) thématise cette continuité en termes de "vases communicants". Salarié depuis plus de 15 ans dans un grand groupe du secteur de l'énergie, il s'en détache quand le produit, tourné vers l'isolation énergétique, lui semble céder le pas à une logique financière. II rejoint alors un groupe activiste après une Marche pour le climat: "je crois dans le produit, je croyais plus en [l'entreprise] en tant que telle, et donc y'avait un gros vase communicant, toute l'énergie que je pouvais avoir, je la mettais dans le [nom du collectif]". Après un passage à vide, il obtient une rupture conventionnelle et devient, à titre alimentaire, consultant externe pour son précédant employeur, tout en élargissant son activité à d'autres industries via une formation: "en montant ma propre structure, en travaillant pour moi, de la manière dont je l'entends, avec les gens que je veux, et surtout en me préparant une activité qui est plus au niveau climatique, de bilan carbone et d'aide aux entreprises, pour avoir le plus d'impact possible". La bifurcation vers l'engagement associatif n'en est pas moins devenu irréversible, dans ce qui s'énonce comme une recherche d' "alignement": "maintenant je ne reviendrais pas en arrière, c'est super intéressant le côté association, mais je réaligne aussi mon bureau pour me focaliser sur ça". II s'engage ainsi dans la transformation climatique de son habitat, en même temps que son activité associative de sensibilisation aux enjeux climatiques s'emploie à "aller chercher le citoyen où il est", à l'université, en entreprise, dans les collèges etc. Le cas est proche de celui d'un autre enquêté, qui tient son activité associative, par la continuité des efforts qu'il y déploie et l'implication temporelle qu'elle engage, comme "quasiment une activité professionnelle".

La tendance à prêter une inégale dignité politique aux "écogestes" et aux mobilisations collectives ou au lobbying politique manquerait le sens, mais aussi la dynamique de cet activisme du quotidien, en laissant hors champ les apprentissages, les effets d'engrenage et les décloisonnements inhérents à un processus où se co-transforment personnes et milieux. Une enquêtée en fait même une conviction:

Je crois que tous les niveaux sont nécessaires, à la fois ce qu'on fait au quotidien, et qui vient planter ses racines, et vraiment infuser autour, l'aspect de ce que tu fais dans ton métier, l'aspect politique local, l'aspect politique général, on fait beaucoup de lobbying citoyen, avec le collectif, on essaye d'informer pour faire changer les pratiques. [...] En fait, tu peux tout à fait retourner les choses. 
Alors, ce n'est pas suffisant, et cela ne va pas du tout assez vite, mais pour moi c'est la seule façon de faire, attaquer à tous ces niveaux là en même temps.

Cette vision continuiste, où activisme du quotidien et engagement multiforme se confondent, les amène aussi à tenir à distance les figures, souvent croquées, du "militant classique, vindicatif", "agressif", ou de "l'écolo qui a toujours raison sur tout".

\section{Transformer ses habitudes de vie et habiter un monde commun, même combat}

À rebours d'une posture de surplomb, les enquêté.e.s valorisent, vis-à-vis des proches comme des inconnus, et jusque dans les activités de sensibilisation, ce qu'ils désignent comme une "posture basse", ou "plus relax", n'aspirant pas (ou plus) à convaincre ou à cliver. De même qu'ils manifestent leur méfiance face à tout "entre soi", ils multiplient les marques de compréhension à l'égard de ceux ne partageant pas leur "mode de vie" ou ayant "moins le temps de se remettre en cause": "Je comprends encore que certaines personnes veulent voyager dans le monde, moi j'en ai plus besoin aujourd'hui, mais je comprends aussi ça"; "j’ai été critique, maintenant je me dis acceptation!". Bien sûr, cela ne les empêche nullement d'éprouver un vif dégoût dans certaines circonstances, mais l'indignation reste tue et les "débats sans fin", soigneusement évités (sauf au sein des couples, où les "clashs" entre co-équipiers sont aussi rares qu'intenses):

\footnotetext{
On s'était retrouvé pour une 'réunion de travail' dans une crêperie en l'occurrence, et une des copines arrive, et se prend une crêpe au nutella, et j'étais hyper choquée, je ne lui ai rien dit. Genre: 'mais tu manges encore du nutella! [rire]. Mais qu'est-ce qu'il faut pour te faire comprendre?' Je ne lui ai rien dit. Je me suis trouvée fantastique. Mais oui, effectivement, j'étais hyper choquée, parce que la plupart des gens de mon groupe ont complètement abdiqué ce genre de trucs.
}

Et de se décrire comme eux-mêmes "incohérents sur plein de points", et sensibles à l'inconfort de se frotter, fût-ce avec méthode, à ce qui éprouve: "c'est dur", "c'est compliqué", "ce n'est pas facile tous les jours" etc. L'avion figure souvent parmi les habitudes les plus difficiles à réviser, même si des avancées sont aussi soulignées ("c'est la première année où on commence vraiment à dire non"):

Donc là y'a eu une réflexion, quand je vais voir ma copine, et hop je prenais l'avion, c'était Easyjet en l'occurrence, comme un taxi, 
je faisais même plus attention aux horaires, j'y allais, j'avais totalement intégré le truc, et donc il a fallu déconstruire ça. Et ça a été par étapes.

Y'avait un ingénieur, il faisait beaucoup de vélo et il m'a dit 'écoute, voilà'. Ils n'ont pas insisté trop, et j'ai mis un certain temps à réaliser, c'est là où je dis c'est progressif, j'étais déjà écolo engagé, je prenais encore beaucoup l'avion, je pense c'est par le mouvement des Villes en Transitions où le sujet a été abordé aussi, je sais plus exactement, et petit à petit je me suis rendu compte que y'avait un problème, 'ah là...'. Et il a fallu que j'ai quand même une petite discussion avec moi-même, ça n'a pas été évident, changer de pratique là-dessus, et même encore parfois aujourd'hui si je suis honnête.

On est à l'opposé de la posture "d'innocence" (Haraway, 2020) qui associe un savoir sur l'effondrement à un rapport dégagé à l'action, dont l'impuissance est simplement actée et attribuée à l'impéritie des autres, comme dans ce post Facebook: "Nous devons diviser nos émissions par huit, ça représente non pas une décroissance mais un effondrement. Nous devons précipiter notre effondrement. Bonne chance pour faire entrer cela dans les consciences. [...] En raccourci, il faudrait aller dans le sens du 'tous comme le Bhoutan' et là on peut commencer à discuter du comment". Le "comment" est au contraire ici d'emblée partie prenante d'une enquête, qui s'emploie à ne pas dénier aux autres la capacité de changer, dont l'expérience est faite pour soi:

Super souvent, quand on fait de la sensibilisation, les gens disent 'nan mais de toute façon les gens ne changeront pas, il faut une dictature écolo', naaaaan [rire]! Déjà arrêtez de dire ça, c'est qui les gens, toi tu changeras? Ouais... Bah voilà! Alors si toi tu changeras, y'a pas de raison que les autres ils ne changent pas, faut arrêter de pas avoir confiance en les gens, ça suffit!

Prendre ainsi parti sur ce que peuvent "les gens", leur plasticité, leur capacité à apprendre de leurs expériences, c'est pouvoir compter sur les interdépendances, c'est-à-dire sur les effets indirects ou ce que J. Dewey appelle le "collateral learning" (Bidet, 2020):

Pour moi y'a un vrai rôle de l'exemplarité, c'est à dire que je le vois, même dans ma classe, avec mes petits pochons, enfin là je ne les ai pas là, mais mes pochons de coton, avec mes, avec ma poire dedans, ou mes Tupperware, enfin c'est ... ou ma gourde, bah ça, ça fait parler. C'est difficile de dire à une personne 'bah nan t'as tort de faire ça, t'achète plus de plastique c'est quand même bizarre', alors que face à un parti politique c'est tellement plus fa- 
cile de critiquer un programme; tu vois ce que je veux dire? Alors que si t'incarne vraiment, dans tes pratiques, ouais là je pense tu touches quelque chose là.

Ainsi, il s'agit de pousser sans pousser. Si on "ne peut pas pousser", car à la fois "ça ne marche pas" et "c'est le choix de chacun", "chacun est libre", on considère néanmoins qu' "agir à côté de manière différente, ça ça pousse": "quand tu changes tes habitudes, ça les affecte". Et de thématiser l'effet "tâche d'huile", éprouvé aussi pour eux-mêmes, de ce qui "infuse ton quotidien au fur et à mesure, plante ses racines". La suggestion est parfois plus directe, dans un moment où l'on se dit alors "particulièrement militant": "bon, allez, ce midi, j'ai récupéré des bocaux, tout le monde part avec ses bocaux, on va chercher à manger comme ça' [rires]. Parfois ça marchait. Bon, je n'ai pas la foi de le faire tous les midis, mais y'avait une démarche qui commençait chez certains".

Cet activisme du quotidien - apparent oxymore - contrarie la disjonction entre éthique et politique souvent postulée par les critiques de l'effondrisme, qui tendent à rabattre les "petits gestes" du quotidien et les "transformations locales" des "effondrés" sur un "discours de moralisation" ou de "culpabilisation individuelle" dé-

9. Cet extrait de post Facebook peut l'illustrer: "L'ascétisme et le souci de soi ont toujours été la politique de l'autruche des âges impériaux. Tes toilettes sèches et ton potager permaculturé ne changeront pas le monde buddy! Par contre, l'action publique, l'investissement syndical et associatif, le rapport de forces sont les signes sains d'une réelle transformation" (apud Bidet, 2019). politisant ${ }^{9}$. Ce faisant, ces discours médiatiques ignorent que le politique peut aussi se lire dans la difficulté à se défaire de gestes, dont les habitudes engagent des visées, des idéaux ou même des idéologies métabolisées de longue date par nos sociétés; comme il se lit dans le travail pour en élaborer de nouveaux et dans la façon même d'interagir avec "les gens" avec lesquels produire les irréversibilités propices à d'autres "formes de vie" - une expression par laquelle J. Dewey définit, à travers la démocratie, la communauté idéale (Bidet \& Gayet-Viaud, 2020).

\section{Conclusion.}

\section{L'activisme du quotidien, aux marges de l'effondrisme?}

Les activistes ou aspirants activistes considérés ici relèvent des publics de l'effondrement, ont des moments effondristes, mais ne correspondent pas pleinement à la skolê effondriste documentée par C. Tasset. La logique d'auto-affectation par la connaissance, relevée sur des terrains explicitement "effondristes" (Tasset, 2020, cet ouvrage), cèdent en effet le pas à une dynamique d'“auto-affectation par l'action", où l'émotion de créer - ou passion, si l'on veut marquer son caractère entretenu (Roux, Charvolin \& Dumain, 2009: 373) prime sur la passion de savoir. L'engagement des enquêté.e.s dans la révision de leur "mode de vie" et la sensibilisation à l'écologie s'alimente à sa propre félicité, à mesure qu'il rend tangible à leurs yeux un "nouveau monde". 
Ce "nouveau monde" est éprouvé au plus intime comme une transformation de soi, mais aussi, jusque dans les espaces les plus publics, comme une contamination du milieu environnant. Ainsi, les entretiens témoignent de quêtes de fidélité à soimême, ponctuées de "transformative moments" (Hards, 2012), de "chocs moraux" (Traïni, 2011), de dynamiques exploratoires (Bidet \& Boutet, 2013), de "bifurcations" (Negroni, 2005), où les intéressés (re)découvrent des attachements "viscéraux", aspirent à se défaire d' "étiquettes" accolées par des tiers et font prévaloir des épreuves de convenance personnelle ("je me sens mieux", "ça me convient", "c'est pas que j'essaye, c'est qui je suis en fait"). Simultanément, la transformation de ses habitudes de vie, comme le plaisir pris à sa narration, partie prenante d'une publicisation, manifeste un "esprit public" au sens de N. Eliasoph (2010), c'est-àdire une orientation vers un monde commun ${ }^{10}$ : on compte sur les connexions, on se soucie des interdépendances, on éprouve l'efficace de l'exemple, on parie sur la réciprocité, on cultive le principe de symétrie, on ménage la responsabilité d'autrui etc. L'activisme du quotidien, comme enquête continuée et pervasive, associe ainsi à une totalisation de l'existence, tournée vers la catastrophe écologique, le souci de cultiver la capacité de chacun à y répondre.

Peut-on séparer ce qui fait expérience, au sens fort du terme, de l'exploration in situ de formes désirables de coexistence - ce processus où se transforme continûment la culture politique d'une société (Bidet et al., 2015; Gayet-Viaud, Bidet \& Le Méner, 2019)? Si l'on peut en douter, l'attention aux moments effondristes amène alors à poser de façon plus ouverte la question de la portée politique de l'effondrisme, en la réinscrivant plus largement dans nos efforts pour hériter de notre monde (Bonnet, Landivar \& Monnin, 2021).

\section{Références}

ALLARD, Laurence; MONNIN, Alexandre; TASSET, Cyprien. Est-il trop tard pour l'effondrement? Multitudes, n. 76, p. 53-67, 2019.

ALLARD, Laurence. Les vulnérabilisé.es volontaires du climat: narrations de soi collapsologistes sur les réseaux socio-numériques. Signes, discours, société. "Dynamiques discursives de la vulnérabilité", n. 76, p. 53-67, Sep. 2019.

BARKER, Kezia. How to survive the end of the future: Preppers, pathology and the everyday crisis of insecurity". Transactions of the Institue of British Geographers, v. 45, n. 2, p. 483-496, 2019.
10. Cette

articulation entre "transformation de soi" et engagements collectifs est aussi attestée in Chamel (2019). 
BIDET, Alexandra. Considérer la fragilité, composer un monde". Faire en citoyen l'expérience du flux des passagers du métro. In: GEFEN, Alexandre; LAUGIER, Sandra (Dirs.). Le pouvoir des liens faibles, p. 27-48. Paris: Éditions du CNRS, 2020.

Faut-il "avertir de la fin des temps pour exiger la fin des touillettes'? Les ressorts de l'engagement écocitoyen. Multitudes, n. 76, Dossier "Est-il trop tard pour l'effondrement?", p. 134-141, 2019.

BIDET, Alexandra; GAYET-VIAUD, Carole. Des écrits politiques de John Dewey à une ethnographie de la citoyenneté comme "vie commune". Pragmata. Revue d'Études Pragmatistes, n. 3, à paraître, 2020.

BIDET, Alexandra; BOUTET, Manuel; CHAVE, Frédérique, GAYET-VIAUD, Carole, LE MÉNER, Erwan. Publicité, sollicitation, intervention. Pistes pour une étude pragmatiste de l'expérience citoyenne. Sociologies, "Dossier Pragmatisme et sciences sociales: explorations, enquêtes, expérimentations", mis en ligne le 23 février 2015.

BIDET, Alexandra; BOUTET, Manuel. Pluralité des engagements et travail sur soi. Le cas de salariés ayant une pratique ludique ou bénévole. Réseaux, La Découverte, v. 6, n. 182, p. 119-152, 2013.

BONNET, Emmanuel; LANDIVAR, Diego; MONNIN; Alexandre; ALLARD, Laurence. Le design, une cosmologie sans monde face à l'anthropocène. Sciences du Design, v. 2 , n. 10, p. 97-104, 2019.

CHAMEL, Jean. Faire le deuil d'un monde qui meurt. Quand la collapsologie rencontre l'écospiritualité. Terrain. Anthropologie \& Sciences Humaines, Apocalypses, n. 71, p. 68-85, 2019.

CHARBONNIER, Pierre. Splendeurs et misères de la collapsologie. Comment rendre l'effondrement désirable. Revue du Crieur, v. 13, Juin 2019.

ChATEAURAYNAUD, Francis; DEBAZ, Josquin. Aux bords de l'irréversible. Sociologie pragmatique des transformations. Paris: Petra, 2017.

CRAVATE,Jérémie. L'effondrement parlons-en... Les limites de la collapsologie. Barricade, 2019. Disponível em: <https://www.barricade.be/sites/default/files/publications/pdf/2019_etude_l-effondrement-parlons-en_1.pdf>.

DEWEY, John. La quête de certitude. Une étude de la relation entre connaissance et action. Paris: Gallimard, 2014 [1929].

. La formation des valeurs. Paris: La Découverte, 2011 a.

. Une foi commune. Paris: La Découverte, 2011b. 
DOBRÉ, Michelle. L'Écologie au quotidien. Éléments pour une théorie sociologique de la résistance ordinaire, Collection "Sociologies et environnement". Paris: Harmattan, 2002.

ELIASOPH, Nina. L'évitement du politique. Comment les Américains produisent l'apathie dans la vie quotidienne. Economica, Collection "Études sociologiques", 2010 [1998].

FITZGERALD, F. Scott. L'effondrement. Paris: Rivages poche, 2011 [1936].

FLAHAULT, François. Le crépuscule de Prométhée. Contribution à l'histoire de la démesure humaine. Paris: Mille et une Nuits, 2008.

GAYET-VIAUD, Carole; BIDET, Alexandra; LE MÉNER, Erwan. Enquêter sur la portée politique des rapports en public. Politix, n. 125, p. 7-30, 2019.

HALKIER, Bente, A practice theoretical perspective on everyday dealings with environmental challenges of food consumption. Anthropology of food, Can consummers save the world?, Sep. 2009.

HARAWAY, Dona. Vivre avec le trouble. Vaulx-en-Velin, FR: Les Éditions Des Mondes à Faire, 2020 [2018].

HARDS, Sarah. Tales of transformation: the potential of a narrative approach to pro-environmental practices, Geoforum, n. 43, p. 760-771, 2012.

LATOUR, Bruno. Où atterrir? Comment s'orienter en politique. Paris: La Découverte, 2017.

MÈGE, Arnaud. "Faire autrement". Tensions entre idéaux et contraintes pratiques de militants pour la décroissance. Terrains \& travaux, v. 31, n. 2, p. 63-86, 2017.

"Une vie qui nous convient". La décroissance, "c'est aussi pour le bien-être de la planète et pour soi aussi... c'est un mieux-être". Savoir/Agir, v. 14, n. 4, p. 5763, 2010.

MONNIN, Alexandre; ALLARD, Laurence. Ce que le design a fait à l'Anthropocène, ce que l'Anthropocène fait au design. "Introduction du dossier 'Anthropocène et effondrement'". Sciences du Design, v. 11, n. 1, p. 21-31, 2020.

NEGRONI, Catherine. La reconversion professionnelle volontaire: d'une bifurcation professionnelle à une bifurcation biographique. Cahiers Internationaux de Sociologie. n. 119, p. 311-331, 2005. 
OLLITRAULT, Sylvie. Militer pour la planète. Sociologie des écologistes, Collection "Res publica". Rennes, FR: Presses Universitaires de Rennes, 2009.

PRUVOST, Geneviève. Chantiers participatifs, autogérés, collectifs: la politisation du moindre geste. Sociologie du Travail, n. 57, 2015.

L'alternative écologique. Vivre et travailler autrement. Terrain, n. 60, "L’imaginaire écologique", p. 36-55, 2013.

RAFANELL I ORRA, Josep. Fragmenter le monde. Contribution à la commune en cours. Paris: Éditions Divergences, 2018.

ROUX, Jacques; CHARVOLIN, Florian; DUMAIN, Aurélie. Les "passions cognitives" ou la dimension rebelle du connaître en régime de passion. Revue d'Anthropologie des Connaissances, v. 3, n. 3, p. 369-385, 2009.

SARNOWSKI, Solène. Les ressorts de l'engagement écologique: enquête sur la formation et la transformation des activités. Mémoire de Master 1 de Sociologie, EHESS, Paris, 2020.

SEMAL, Luc. Face à l'effondrement. Militer à l'ombre des catastrophes, Collection "L'écologie en questions". Paris: Presses Universitaires de France, 2019a.

Le catastrophisme fait partie de l'écologie politique. Entretien avec Hugo Struna, Usbek \& Rica, 30 Mai 2019b.

STENGERS, Isabelle. William James: une éthique de la pensée. In: DEBAISE, Didier (Dir.). Vie et expérimentation: Peirce, James, Dewey. Paris: Vrin, 2007.

SZUBA, Mathilde; SEMAL, Luc. Rationnement volontaire contre "abondance dévastatrice": I'exemple des crags. Sociologies Pratiques, v. 20, n. 1, p. 87-95, 2010.

TASSET, Cyprien. L'effondrisme comme passion de savoir. "Puissance d'expression et réalisme épistémique". Document de travail, 2020.

Les "effondrés anonymes"? S'associer autour d'un constat de dépassement des limites planétaires. La Pensée Écologique, v. 1, n. 3, p. 53-62, 2019.

TRAIINI, Christophe. L'enfance et les sensibilités primordiales de la lutte pour la protection animale. In: MUXEL, Anne (Dir.). La politique au fil de l'âge, p. 213-228. Paris: Presses de Science Po, 2011. 